\title{
The person-oriented approach: A short theoretical and practical guide*
}

\author{
Lars R. Bergman ${ }^{\mathrm{a} 1}$, Margit Wångby ${ }^{\mathrm{b}}$ \\ a Stockholm University \\ ${ }^{b}$ Lund University
}

\begin{abstract}
A short overview of the person-oriented approach is given as a guide to the researcher interested in carrying out person-oriented research. Theoretical, methodological, and practical considerations of the approach are discussed. First, some historical roots are traced, followed by a description of the holisticinteractionistic research paradigm, which provided the general framework for the development of the modern person-oriented approach. The approach has both a theoretical and a methodological facet and after presenting its key theoretical tenets, an overview is given of some common person-oriented methods. Central to the person-oriented approach is a system view with its components together forming a pattern regarded as indivisible. This pattern should be understood and studied as a whole, not broken up into pieces (variables) that are studied as separate entities. Hence, usually methodological tools are used by which whole patterns are analysed (e.g. cluster analysis). An empirical example is given where the pattern development of school grades is studied.
\end{abstract}

Keywords: person-oriented, individual development, longitudinal, school grades

\section{Introduction}

Before modern scientific psychology emerged, psychology was a very different discipline, if it at all existed. For instance, what we today call "psychology" was often regarded as a part of philosophy and was much less oriented towards empirical research and the use of quantitative methods (Boring, 1950). In the "old days" a holistic approach was rather common in which a person was regarded as a totality and, for instance, seen as belonging to one of a number of types, each characterised by a set of dominant features. This goes back to the old Greeks; for instance

Version February 16, 2014.

1 Department of Psychology, Stockholm University, 10691 Stockholm, Sweden;

lrb@psychology.su.se 
the Hippocratic school, especially Galen, claimed that the mix of the four humors (blood, black bile, yellow bile and phlegm) decided which of four types of temperaments characterised a person: "sanguine", "choleric", "melancholic" or "phlegmatic". (Kagan, 1998). Before modern scientific psychology, the typological approach was characterised by subjectivity and a lack of empirical research that hampered scientific progress and created a plethora of often ill-founded typologies. Of course, a holistic "system view" has for hundreds of years been expressed by scientists in other disciplines, for instance by Carl von Linné (1978), the pioneer of "modern" taxonomy in biology.

Beginning in the $19^{\text {th }}$ century a new type of psychology emerged, largely based on the successful research methods developed within the natural sciences in which empirical research, experiments, quantification, and replication of findings were stressed. The variable became a central conceptual and analytical unit, allowing for a decomposition of reality into different dimensions and a structured and detailed study of psychological phenomena, and this was facilitated by newly developed powerful statistical methods. This variable-oriented research paradigm led to a knowledge explosion in psychology and contributed to discrediting the typological approach as largely subjective and primitive.

However, during the last few decades and inspired by the modern physics, an interactionistic theoretical framework has grown stronger in psychology in which the individual is regarded as a dynamic system of interwoven components that is best understood in terms of whole-system properties (e.g. Magnusson, 1988). Seen from this perspective, in many research settings there is a mismatch between the standard variableoriented approach that is applied and the dynamic process view now held by many scholars (Bergman \&Vargha, 2013). There is also to some extent a correspondence between the modern whole-system view and the thinking behind many old typological theories. Based on an interactionistic framework, a modern person-oriented approach has emerged with one pioneer being Jack Block (Block, 1971). In this approach, the individual is seen as a functioning totality, often best studied by analysing patterns of information, not separate variables, and by searching for typical patterns, each being approximately shared by a subgroup of the total sample. To some extent the approach can be regarded as "typological" but, in contrast to the old typological approach, it lends itself to quantification, replication, and it allows procedures for theory testing and falsification. The similarity to the old typological approach is limited to the basic view 
that the person should as far as possible be studied as an organised whole (see Bergman, Andershed, \& Andershed, 2009).

In the present article, a short overview is given of the modern personoriented approach. The purpose is to provide the reader with a guide to see the possibilities and limitations of the approach in relation to his/her own research area. For technical details and more in-depth discussions of various issues, the reader is referred to the references we give.

\section{Theoretical considerations}

The modern person-oriented approach is presented in Bergman and Magnusson (1997) and in Bergman, von Eye, and Magnusson (2006). It should be noticed that the approach has two facets: (1) a theoretical facet and (2) a methodological facet in the form of methods that most naturally are useful in implementing person-oriented research (usually patternoriented methods).

In the holistic-interactionistic research paradigm the individual is seen as an organised whole with elements operating together to achieve a functioning system in a dynamic process with interactions between components. Components could be of different kinds, for instance, behaviours, biological factors, environmental factors, and so on (Magnusson, 1988; Magnusson \& Törestad, 1993). This view is related to a dynamical systems view that exists in many other sciences (see e.g., Kelso, 2000; Devaney, 1989). The paradigm can be applied to almost all studies of individuals, guiding problem formulation, research strategy and methodology, and guiding how to interpret findings. The paradigm can also function as a lingua franca for communication between researchers in different fields of psychology and between psychology and neighbouring sciences (Magnusson, 1996). Many basic theoretical conceptualisations of the paradigm are not unique but are shared by a number of developmental theoreticians, for instance Bronfenbrenner and Evans (2000) and are incorporated in the new developmental science (Cairns, Elder, \& Costello, 1996; Cicchetti 2006).

The theoretical conceptualisations of the person-oriented approach are rooted in the holistic-interactionistic research paradigm and its basic tenets are presented in Bergman and Magnusson (1997). Below we present four of these tenets in a modified form. 
(1) The conceptual model of the developmental process is the one proposed by the holistic-interactionistic research paradigm. Hence, the process is partly individual-specific.

(2) There exists a hierarchy of process levels (e.g. the system of selfexperienced factors operating in an individual during test-taking is subsumed under the system of individual characteristics interacting with the situational context). There is coherence and structure in the development and functioning of the systems at the different levels.

(3) The developmental process follows laws that relate to structures organised and functioning as patterns of operating factors. These laws are assumed to have communalities across individuals but they cannot be assumed to be identical across individuals.

(4) In the developmental process, "typical patterns" of observed system components often emerge both within the individual and across individuals. Bergman and Magnusson (1997, p. 293) express this as follows. "Although there is, theoretically, an infinite variety of differences with regard to process characteristics and observed states at a detailed level, there will often be a small number of more frequently observed patterns ("common types"), if viewed at a more global level." They present support for this assumption and for why it should be tested on empirical data. The postulated "typical patterns" have similarities to the "attractors" studied in dynamical systems research.

\section{Methodological considerations}

The person-oriented theoretical view has implications for the choice of research methodology in empirical research. Two important implications are the following:

(1) The methodology should, at least to a certain extent, allow for inferences about the single person. As pointed out by, for instance, Molenaar (2004) and von Eye and Bergman (2003), common variableoriented methods applied to inter-individual empirical data do not normally fulfil this requirement. To give a simple example: A stability coefficient is computed as a Pearson correlation between a pretest measure and a posttest measure of the same variable. If the correlation is reasonably high (say 0.60 ) the interpretation often is that "an individual who starts low tends to end up low and an individual who starts high tends to end up high". However, in this case with an explained variance of $0.60^{2}=0.36$, this statement is not generally true at the indi- 
vidual level. There is a substantial proportion of the individuals who follow a different developmental track, for instance they start above the average and end up below the average.

(2) The methodology should allow for inferences about individual patterns of functioning. This can normally only be accomplished by treating the key pattern defining the system of interest (usually a vector of variable values) as an indivisible unit in the analysis. To give an example: Consider a data set with just three binary variables describing the system of interest. Further, consider the case where all pairwise relationships among the three variables are zero. Now, these data contain $2 \times 2 \times 2=8$ possible value patterns and it is possible that they are present in frequencies so that there are strongly significant typical patterns - in spite of the fact that all pairwise relationships are zero. The researcher ignoring information at the pattern level and only analysing pairwise relationships would then falsely assume there is no interesting structure in the data. This example is called "Meehl's paradox" (Meehl, 1950) and it illustrates the fact that it cannot automatically be assumed that a correlation matrix or a variance-covariance matrix contains all relevant information about the relationships that exist in a data set. And yet, in standard applications of structural equation modelling, for instance, this assumption is made and model fit is measured by the ability of the statistical model to reproduce the correlation matrix. Especially from a person-oriented perspective, such an assumption is not well-founded because there might be typical patterns in data (akin to higher-order interactions) that are not mirrored by a correlation matrix of pairwise relationships.

\section{Some common methods used in person-oriented empirical research}

\section{The study of the single individual}

One approach in person-oriented research is the study of the single individual (e.g. Molenaar, 2004; Nesselroade \& Ford, 1985). First, single individuals are studied separately, usually based on data from a large number of measurement occasions. Then attempts are made to generalise the findings across individuals using a "bottom-up" approach. In one sense, this approach is truly person-oriented because single persons are at focus and are studied intensively. In another sense it is usually not personoriented because variables are analysed, not patterns 


\section{Exploratory classification analysis based on patterns of information}

Cluster analysis is often used to perform exploratory classification analysis with the aim to find, usually non-overlapping, clusters/groups ("typical patterns") where the members in the same cluster have similar value patterns. If cross-sectional data are analysed, the approach is personoriented in the sense that the pattern and to some extent the individual is at focus. However, the process aspect of the person-oriented approach is ignored with a cross-sectional approach. This aspect is to a certain extent incorporated in cluster analysis-based methods where longitudinal data are analysed. One such method is Linking of Clusters after Removal of a Residue - LICUR (Bergman, Magnusson, \& El-Khouri, 2000). In LICUR, separate cluster analyses are performed on a set of variables at two or more measurement occasions and then the resulting classifications are linked. In this way, structural and individual stability and change can be studied at the pattern level. Another method is I-States as Objects Analysis - ISOA (Bergman, Nurmi, \& von Eye, 2012). In ISOA, shortterm development in a set of variables is studied by first finding a timeinvariant classification system, usually by employing cluster analysis. Then the sequences of the individuals' cluster memberships in the invariant classification system are studied across measurement occasions.

\section{Studying all possible value patterns "directly"}

Applying complex statistical methods for analysing patterns tends to create a large distance to the data being analysed: they are seen through a complex lens. Hence, there is a need for a method that helps the researcher to see and easily understand more directly the structures that appear in the data. This need has led to the development of Configural Frequency Analysis - CFA (Lienert \& Krauth, 1975; von Eye \& Pena, 2004). The basic idea in CFA is that first all variables in the pattern are categorised into a few categories (usually two or three) and then all possible patterns are listed. It is noted how frequent each pattern is and if it is a type, i.e. occurs significantly more often than expected by a base model, or an antitype, i.e. occurs significantly less often than expected. CFA has grown into a family of methods that can be applied for analysing very different types of data, for instance longitudinal data. 


\section{Model-based classification analysis}

A statistical model is estimated that contains a latent categorical variable that "explains" the relationships in the data and the model's parameters are estimated from the sample. This means that confidence intervals can be given and model fit tested. Variants of this approach are latent class analysis for analysing categorical data (Lazarsfeld \& Henry, 1968) and latent profile analysis for analysing continuous data (Gibson, 1959; Muthén, 2002). These methods have been expanded to allow the study of development, for instance, in latent transition analysis categorical data can be subjected to longitudinal analysis (Collins \& Wugalter, 1992). If all essential assumptions are approximately fulfilled, a model-based approach can be preferable - but the power to reject a bad model can be low for moderate sample sizes. For a discussion of the pros and cons of an exploratory versus model-based classification analysis, the reader is referred to Sterba and Bauer (2010).

\section{Models for studying nonlinear dynamic systems}

Models for studying nonlinear dynamic systems - NOLIDS (Barton, 1994; Boker, 2002; Granic \& Hollenstein, 2006) is an import from the natural sciences and the aim is to find a mathematical model of how a process evolves. This is usually accomplished by finding a set of differential equations that model change in the system's behaviour across time. In one sense, NOLIDS is a truly person-oriented approach since wholesystem development is at focus. However, these kinds of models can be hard to implement in many contexts in psychology: their ideal application is in a controlled experiment with very many measurement points. Nevertheless, for the person-oriented researcher NOLIDS have many attractive properties, even if the actual model building can be extremely difficult in many cases. For instance, many NOLIDS concepts, new to most psychologists, can be useful as aids in one's theoretical thinking (e.g. "attractor", "chaos", "slaving", etc.).

For more extensive overviews of person-oriented methods the reader is referred to Bergman et al. (2000), Bogat, von Eye, and Bergman (in press), Sterba and Bauer (2010), and von Eye and Bergman (2003). 


\section{Empirical example: \\ The development of school grades between the age of 10 and 13}

Considering the topic of this issue of the journal, an example is given from the field of educational psychology. To illustrate the essence of the person-oriented approach in as simple a form as possible, the example is chosen so that it is not necessary to use any advanced methodology and a minimum of variables are analysed.

The data were taken from the Swedish longitudinal programme Individual Development and Adaptation - IDA (Magnusson, 1988). Data concerning school grades in Swedish and Mathematics were analysed for about 1,000 children, with the same children measured twice, namely, at age 10 and age 13 . The school grades are scaled $1-5$ with " 5 " being the highest grade. Different teachers did the grading for each age group.

Some background information from simple variable-oriented analyses is presented in Table 1.

Table 1. Correlation matrix, means, and standard deviations for school grades at age 10 and age 13

\begin{tabular}{l|c|c|c|c|c}
\hline & Swedish10 & $\begin{array}{c}\text { Mathe- } \\
\text { matics10 }\end{array}$ & Swedish13 & $\begin{array}{c}\text { Mathe- } \\
\text { matics13 }\end{array}$ & Mean/sd \\
\hline Swedish10 & 1 & .66 & .75 & .59 & $3.1 / 1.0$ \\
\hline Mathematics10 & & 1 & .61 & .72 & $3.2 / 1.0$ \\
\hline Swedish13 & & & 1 & & $3.1 / 0.9$ \\
\hline Mathematics13 & & & & 1 & $3.2 / 1.0$ \\
\hline
\end{tabular}

Note. Swedish10 is the grade in Swedish at age 10. Using multiple linear regression, Swedish 13 and Mathematics 13 were predicted by both grades at age 10 and $R^{2}$ was .58 and .54 , respectively. Both grades were significant predictors but the strongest one was the same grade at age 10 that was the dependent variable at age 13.

It is seen in Table 1 that the correlation between the school grades in Swedish and Mathematics is rather high at both ages and that their stability coefficients are high. The means and standard deviations are similar at both ages. A school grade also correlates very highly with the corresponding standardised achievement test (above 0.80, not shown in Table 1). Both grades are well predicted by the two grades measured three years earlier with explained variances somewhat above $50 \%$.

In Table 2 the relative frequencies of the $5 \times 5=25$ possible grade patterns at a specific age are given for age 10 and age 13 separately. For instance, as expected, it is seen that the most common grade pattern is 33 , 
both at age $10(21.2 \%)$ and at age $13(21.4 \%)$. It is also seen that having an identical grade pattern at age 10 and age 13 is in many cases much more common than expected by chance, being most strongly expressed for those with an even grade pattern. For instance, $46.4 \%$ of children starting with the grade pattern 55 show the same pattern at age 13, which is 16.3 times more often than expected by chance.

Table 2. Relative frequency (\%) of grade patterns in Swedish and Mathematics at age 10 and at age 13

\begin{tabular}{|c|c|c|c|}
\hline $\begin{array}{l}\text { Grade pattern } \\
\text { (even patterns } \\
\text { in bold type) }\end{array}$ & $\begin{array}{c}\text { Relative } \\
\text { frequency (\%) } \\
\text { Age } 10\end{array}$ & $\begin{array}{c}\text { Relative } \\
\text { frequency (\%) } \\
\text { Age } 13\end{array}$ & $\begin{array}{l}\text { Percentage showing } \\
\text { significant* individual } \\
\text { stability of grade pattern }\end{array}$ \\
\hline 11 & 1.1 & 1.2 & \\
\hline 12 & 2.8 & 2.1 & \\
\hline 13 & 0.7 & 0.2 & \\
\hline 14 & 0.0 & 0.0 & \\
\hline 15 & 0.0 & 0.0 & \\
\hline 21 & 1.6 & 2.7 & \\
\hline 22 & 9.3 & 11.5 & 39.8 ( x 3.2) \\
\hline 23 & 9.0 & 6.9 & $28.7(x 4.2)$ \\
\hline 24 & 1.2 & 1.1 & \\
\hline 25 & 0.1 & 0.0 & \\
\hline 31 & 0.4 & 0.7 & \\
\hline 32 & 6.8 & 8.0 & $30.5(x 3.8)$ \\
\hline 33 & 21.2 & 21.4 & 39.4 ( x 1.8) \\
\hline 34 & 9.1 & 8.1 & $24.1(x 3.3)$ \\
\hline 35 & 0.5 & 1.2 & \\
\hline 41 & 0.1 & 0.0 & \\
\hline 42 & 1.1 & 0.9 & \\
\hline 43 & 7.2 & 7.0 & $27.7(x 3.8)$ \\
\hline 44 & 15.6 & 13.6 & $40.4(\times 3.1)$ \\
\hline 45 & 4.0 & 6.9 & $45.9(\times 6.5)$ \\
\hline 51 & 0.0 & 0.0 & \\
\hline 52 & 0.0 & 0.0 & \\
\hline
\end{tabular}




\begin{tabular}{c|c|c|c}
\hline $\begin{array}{c}\text { Grade pattern } \\
\text { (even patterns } \\
\text { in bold type) }\end{array}$ & $\begin{array}{c}\text { Relative } \\
\text { frequency (\%) } \\
\text { Age 10 }\end{array}$ & $\begin{array}{c}\text { Relative } \\
\text { frequency (\%) } \\
\text { Age 13 }\end{array}$ & $\begin{array}{c}\text { Percentage showing } \\
\text { significant* individual } \\
\text { stability of grade pattern }\end{array}$ \\
\hline 53 & 0.9 & 0.6 & \\
\hline 54 & 3.6 & 2.7 & $34.4(\times 12.2)$ \\
\hline $\mathbf{5 5}$ & $\mathbf{3 . 2}$ & $\mathbf{3 . 2}$ & $\mathbf{4 6 . 4}(\mathbf{x}$ 16.3) \\
\hline All & 100.0 & 100.0 & \\
\hline
\end{tabular}

Note. *Bonferroni corrected probability using the hypergeometric distribution with nominal probability set to $0.05 / 25$. For those patterns showing significant stability, the percentage is presented of all having the pattern at age 10 that show the same pattern at age 13 and within parenthesis the ratio is given between observed value and expected value according to an independence model.

A detailed study of individual grade pattern development can be accomplished by studying the cross tabulation between the grade pattern at age 10 and the grade pattern at age 13 . This is a $25 \times 25$ cross tabulation and, if carefully analysed, it provides rich information that can answer a number of questions of a person-oriented nature. However, it is not practical to present and interpret such detailed information within the framework of a short empirical example. Hence, as examples we only present some simple questions that were addressed by examining the $25 \times 25$ cross tabulation:

1. We know that grades are rather stable characteristics but how stable are the grade patterns across three years? Short answer: The grade patterns are extremely stable, $33.0 \%$ have an identical grade pattern at both ages and only $21.1 \%$ change their grade pattern more than minimally (i.e. change more than just a single step in just one of the two subjects).

2. How rare are large improvements/deteriorations in grades? Short answer: Only $2 \%$ increase their grades more than one step in any subject and only $3 \%$ decrease their grade more than one step in any subject.

3. Is there any chance for those with low grades in both Swedish and Mathematics to achieve good grades in both subjects three years later? Short answer: No one out of 141 children with a grade pattern with not more than "2" in both Swedish and Mathematics at age 10 ends up with a grade pattern with at least a " 4 " in both grades.

In conclusion, the results from the basic variable-oriented analyses presented in Table 1 indicate a fairly high degree of stability of the grades 
in Swedish and Mathematics - but the results from the simple personoriented analyses reported above point to a much stronger stability of grades, if data are viewed at a more detailed pattern level. If these findings are replicated on other samples, there may be grounds to say that the chance for a school child to improve strongly his/her school performance is almost nonexistent, unless some powerful intervention is implemented.

\section{Some technical comments to the empirical example}

It should be noted that the analyses performed are a simple form of prediction CFA and that they can be further elaborated using methods developed within that tradition. Central to our analyses is the analysis of single cells in the cross tabulation of age 10 grade pattern against age 13 grade pattern (a $25 \times 25$ table). Cells indicating stability in grade pattern (identical patterns in both grades, 25 cells) were significance tested to see if an observed frequency is significantly larger than expected ("types" in CFA terminology). (In a smaller cross tabulation it might also be interesting to search for cells indicating significant types of change in patterns, i.e. exploring the remaining $625-25$ cells, this is not done here). As 25 tests were performed a Bonferroni correction was applied, demanding a nominal significance at the $0.05 / 25=0.002$ level for the result to be "truly" significant at the 5\% level. In most cases, this kind of test can be carried out by a standard statistical package, for instance by examining the standardised adjusted residuals in SPSS output, which approximately follow a standard normal distribution. However, when the expected frequency is small the normal approximation is not very accurate, and unfortunately quite inaccurate at the extreme tails. In this case an exact significance test is much preferable and the hypergeometric distribution can instead be used. This distribution was used for the present data.

\section{Some practical considerations when carrying out a person-oriented approach}

For the sake of brevity, only two basic situations in person-oriented research will be treated, namely: (1) a researcher attempts to find typical patterns in a sample of persons and information is available for a set of approximately continuous variables that together constitute the value profile to be analysed (Case 1), and (2) a researcher wants to study individual treatment effects (Case 2). 


\section{Case 1}

1. A cardinal task is to define the system of interest and its components. For each component, a variable must be found in the data set that matches it. This first task is the most theoretically demanding in that the researcher must be able to defend his/her choice of variables making up the pattern or profile. For instance, available variables cannot just be added to the profile on the simple principle "the more the better", because a change in the set of variables included in the profile usually means that the system studied is changed. Even the addition of a single variable will usually change the classification structure that comes out of the analysis. This holds even if the added variable is another measurement of a similar concept that is already measured by a variable in the profile. For instance, including two different measures of aggression as separate variables in a profile would mean giving the aggression component "double weight" in relation to the other components in the classification analysis. It is better to form a weighted sum of them and include the sum as one variable in the profile.

2. Once the variables to be included in the profile or pattern have been decided, the next step is the choice of a suitable classification method. Both an exploratory method, like cluster analysis, and a modelbased method, like latent profile analysis, are possible alternatives and it is often helpful to perform both types of analyses and compare findings. On the one hand, most model-based methods make stronger assumptions concerning data properties (e.g. that the variables are normally distributed, and that local independence as defined by the model is "true" local independence) and they are more complex than most exploratory methods. On the other hand, model-based methods produce estimates with confidence intervals, allow for model testing, and "benign" measurement errors are modelled (in most exploratory methods even moderate errors of measurement can distort the findings). If cluster analysis is used, an important consideration that should be guided by theory is the choice of a suitable measure of the dissimilarity between two individual profiles. Often squared Euclidean distance is a suitable measure because it takes into account differences between two profiles in both form and level.

3. There is not room in this short article for discussing the technically complicated model-based methods further and we concentrate instead on the case where an exploratory method is used. However, many of the points we bring up are also of relevance in the model-based case. 
4. Often cluster analysis is used for exploratory classification and a host of different algorithms exists. Agglomerative hierarchical cluster analysis can be a good choice, using, for instance, Ward's method that maximises the explained variance of the cluster solution, or average linkage (UPGMA) that minimises the average distances between members in a cluster. For an overview of clustering methods within a person-oriented context the reader is referred to Bergman et al. (2000). Sometimes a relocation method is applied after a preliminary classification has been obtained with a hierarchical method, to further increase the homogeneity of the clusters. Usually, this is to some extent accomplished, but the hierarchical property of the original solution is lost (in a hierarchical solution, the solution with $\mathrm{k}$ clusters is equal to the solution with k-1 clusters, except for two of the $\mathrm{k}$ clusters were fused into one; this is no longer the case after a relocation procedure).

5. How is a "good" cluster solution to be found and validated? First, it should be made clear that the "task" of a cluster analysis is much more difficult than it is for most variable-oriented methods. Let us assume a researcher has $N=200$ persons measured in $k=7$ variables. In a standard variable-oriented method a data summary is often first extracted in the form of a variance-covariance matrix, based on the assumption that a linear model holds. This matrix contains 28 pieces of information that are considered as sufficient input for subsequent analysis (e.g. for constructing a structural equation model which can be regarded as a model that "explains" the variance-covariance matrix). In contrast, in most types of cluster analysis the data are first summarised by a dissimilarity matrix composed of the dissimilarities between all pairs of persons. In the above case this matrix contains 19,900 pieces of information that are input to a cluster analysis that aims at classifying the persons into clusters so that the within-cluster dissimilarities are as small as possible. Obviously, different methods can be used for this purpose and findings can differ between methods (cf. that even in the simple one-dimensional case, summary measures like the arithmetic mean and the median may differ). The cluster structures usually differ only moderately between sound algorithms but it can be wise to use two different methods and compare the findings. Different criteria exist for deciding what is a "good" cluster solution and a suitable number of clusters, see for instance Bergman et al. (2000) for an overview. Common criteria are theoretical meaningfulness of the cluster structure, homogeneity of the clusters, replication of clusters using a different classification method or using 
a different sample, and that theoretically expected differences between clusters emerge in a validation variable.

6. Most classification methods, where subjects are sorted into classes or clusters based on profiles of values in several variables, have two limitations: (1) Assuming the analysed data set is a sample from a population there will be sampling variation in the findings and especially small clusters in the population that are not "tight" might not be found if a small sample were analysed. (2) When an exploratory method like cluster analysis is used, it is important that the variables in the profile are reliably measured, otherwise outliers created by errors of measurement can distort the findings. In fact, even "true" outliers can have this effect (as in a regression analysis) and it might be preferable to remove them before the analysis (Bergman, 1988).

7. Many of the considerations mentioned above also apply in more complex situations, for instance in developmental studies where longitudinal data are analysed.

8. A number of statistical packages exist that can be used for personoriented analysis. With regard to cluster analysis, the most extensive package is Clustan (2005), but basic cluster analysis can be performed using standard statistical packages like SPSS. With regard to modelbased classification analysis, both cross-sectional and longitudinal analyses can be performed using Mplus (Mplus, 2013) and for categorical variables there is a statistical program that performs latent class and latent transition analysis (LTA, 2013). SLEIPNER (2002) and ROPstat (2011) are two statistical packages especially designed for person-oriented analysis. The last two packages can also perform basic CFA analysis but more advanced CFA analyses can be accomplished by using, for instance, programs developed by von Eye (1998).

\section{Case 2}

1. Let us assume a simple standard type of study of treatment effects where subjects in a sample have been randomised into either a treatment group or a control group. Pretest and posttest measures of the dependent variable have been obtained (e.g. rated phobic symptoms in a study of the effect of CBT therapy). Let us further assume that an ANOVA demonstrated a significant treatment effect with an effect size of $d=0.7$ and that the study was well designed. Then average causality has probably been demonstrated: at a group level the patients profited by the therapy. Here, most studies stop - but what about 
individual causality? In this type of study, even a superficial look at the data usually suggests that not everyone seems to have profited by the therapy. Which persons have not done that? A crude measure of an individual's treatment effect would be the difference between the person's posttest value and the predicted posttest value from the pretest value using a regression equation based on the control group. However, this difference has an uncertainty interval that increases with decreasing reliability of the dependent variable. It is well-known that this uncertainty is uncomfortably large if the reliability is not very high (see e.g. Bergman, 2010 for an example). In our example, and assuming a reliability of 0.80 , this means that for most persons it cannot be decided whether a specific person profited or not by the therapy.

2. The above example illustrates a general principle: To make inferences at the individual level the measurement precision has to be high, higher than it usually is in most contexts in psychology (Bergman \& Andersson, 2010).

3. The frequent lack of high reliability of measurements and the common use of group-based variable-oriented methods have led to a dearth of studies providing information about persons instead of variables in modern psychology. For instance, Carlson (1971) examined a large set of articles in journals publishing personality research. He found almost exclusively information about variables (means for different groups, correlations, etc.), not information about single persons. The title of his article is "Where is the person in personality research?"

\section{Discussion}

Hopefully, the brief presentation we have given of the person-oriented approach has shown that the approach should seriously be considered in empirical research, both with regard to its theoretical framework and with regard to the use of methods that are compatible with this framework. What then are its possible drawbacks? These are discussed in Bergman and Andersson (2010) and can in a modified form be summarised in four points:

(1) The person-oriented approach as a general theoretical framework is not very controversial since many of its basic theoretical conceptualisations probably are believed to be valid by most researchers. However, the theory is quite general and its translation into hypotheses that can be empirically tested can be difficult. One reason is that, within almost every area, the body of scientific knowledge is variable- 
oriented, not person-oriented, and of limited use for specifying a person-oriented theory. The definition of the system to study is also a critical task, especially the choice of an adequate set of variables to represent the system. It is not possible in a single study to incorporate the full complexity of the "person-as-a-whole" and, in practice, a person-oriented study is usually restricted to studying a simplified "small" system at a single level. This is suboptimal but it is better than to completely ignore whole-system properties, which usually is the case in variable-oriented research.

(2) In person-oriented research it is usually appropriate to use methods where the basic unit of analysis is a pattern of variable values. However, analyses based on patterns tend to be more complex than analyses based on variables. For instance, the development of a theory of a sampling distribution can be exceedingly difficult when the involved parameters are complex multivariate entities. Hence, methodological problems can present obstacles to an optimal implementation of a person-oriented approach.

(3) As was previously pointed out, the focus on the individual often demands a high reliability of the measures used. This can be hard to accomplish in practice.

(4) The label "person-oriented" and similar ones (e.g., "person-centred", "pattern-oriented") have been given different meanings. Some researchers even ignore the theoretical parts of the person-oriented approach and define the approach just by the method (e.g. "it is cluster analysis"). This is not really a drawback of the person-oriented approach as presented here, but it creates confusion.

A person-oriented and a variable-oriented approach are sometimes compared with regard to their power to predict an important outcome (Asendorpf, 2003; Bergman \& Trost, 2006). A typical setting is the following: (1) A set of variables is regarded as a pattern and subjected to a cluster analysis, resulting in a number of clusters describing typical patterns that occur in the data. (2) The variables in the pattern are then treated as single variables and they are all entered as predictors of an outcome in a multiple regression analysis. Finally, dummy variables coding for cluster membership are added to the predictors to see if they improve the prediction. This is sometimes the case and sometimes not. Two comments are relevant here. Firstly, a description of multivariate data by cluster membership means an extremely condensed summary (the information content is usually only a single categorical variable 
with 3-10 categorical values). On the other hand, the description of the data by several variables that are reasonably continuous is much less condensed, usually many thousands of value combinations occur. In this light it is surprising that often cluster membership is almost as good for predicting an outcome as the original variables. Occam's razor tells us that this should be considered when comparing the approaches. Secondly, how well a phenomenon is understood cannot only be judged from predictive power, as discussed by Bergman and Magnusson (1997). For instance, most commonly a theory formulated within an interactionistic framework is difficult to test on empirical data using a variable-oriented approach, but it can be tested using a person-oriented approach. An example of this is the study of positive and negative perfectionism by Lundh, Saboonchi, \& Wångby (2008).

Many researchers have pointed to a rather frequent mismatch between interactionistic and dynamic theoretical formulations and the statistical methods used to test these theories on empirical data (Bergman \& Magnusson, 1997; Bergman \& Vargha, 2013; Gottlieb, 1996; Richters, 1997). For instance, linear statistical models are applied that are not matched to the researcher's theoretical conceptualisations, resulting in the findings not being interpretable in relation to the theoretical model. In such situations a person-oriented approach can be a better alternative. When process characteristics are at focus, an attractive alternative can be a nonlinear dynamical systems model, if suitable data are available. The parameters of a successful model of this type often nicely correspond to interactionistic theoretical conceptualisations

It is important to recognise that both a person-oriented and a variableoriented approach, as discussed in this article, have a theoretical and a methodological facet. For instance, in some cases it is possible to carry out a study that is truly person-oriented using variable-oriented statistical methods (e.g. a specific combination of values in the components of a system that is studied is believed to be crucial to the emergence of a certain outcome and this combination is dummy coded and entered as a predictor in a regression analysis together with other variables). Or conversely, within a variable-oriented theoretical framework it might be of interest to check for interactions. With many variables involved this often becomes complex when a large number of interactions have to be tested and then a method like cluster analysis can be helpful as a means to suggest "promising" interactions. However, in most cases a personoriented theoretical framework makes it most appropriate to apply pattern-oriented methodology. Sometimes it is helpful to apply both 
person-oriented and variable-oriented methods, but the types of results they produce differ and are difficult to compare. It can be as if a seeing but deaf person compares his or her understanding of a specific situation with that of a blind but hearing person. A comparison of person-oriented and variable-oriented approaches is given by Laursen \& Hoff (2006).

\section{Acknowledgements}

This study was made possible by access to data from the longitudinal research programme Individual Development and Adaptation (IDA). The scientific leaders of IDA are Henrik and Anna-Karin Andershed, before that Lars R. Bergman and David Magnusson. David Magnusson was responsible for the planning, implementation, and financing of the collection of data used in this study. The data collections and database were supported by grants from the Swedish National Board of Education, the Swedish Committee for the Planning and Coordination of Research, The Bank of Sweden Tercentenary Foundation, and the Swedish Social Research Council.

\section{References}

Asendorpf, J. B. (2003). Head-to-head comparison of the predictive validity of personality types and dimensions. European Journal of Personality, 17(5), 327-346. http://dx.doi.org/10.1002/per.492

Barton, S. (1994). Chaos, self-organisation, and psychology. American Psychologist, 49(1), 5-14. http://dx.doi.org/10.1037/0003-066X.49.1.5

Bergman, L. R. (1988). You can't classify all of the people all of the time. Multivariate Behavioral Research, 23(4), 425-441. http://dx.doi.org/10.1207/s15327906mbr2304_1

Bergman, L. R. (2010). The interpretation of single observational units' measurements. In M. Carlson, H. Nyquist, \& M. Villani (Eds.), Official statistics: Methodology and applications in honour of Daniel Thorburn (pp. 37-49). Stockholm: Stockholm University.

Bergman, L. R., Andershed, H., \& Andershed, A-K. (2009). Types and continua in developmental psychopathology: Problem behaviors in school and their relationship to later antisocial behavior. Development and Psychopathology, 21(3), 975992. http://dx.doi.org/10.1017/S0954579409000522

Bergman, L. R., \& Andersson, H. (2010). The person and the variable in developmental psychology. Journal of Psychology, 218(3), 155-165.

Bergman, L. R., \& Magnusson, D. (1997). A person-oriented approach in research on developmental psychopathology. Development and Psychopathology, 9(2), 291319. http://dx.doi.org/10.1017/S095457949700206X 
Bergman, L. R., Magnusson, D., \& El-Khouri, B. M. (2000). Studying individual development in an interindividual context: A person-oriented approach. Mahwah, NJ: Erlbaum.

Bergman, L. R., Nurmi, J-E., \& von Eye, A. A. (2012). I-states-as-objects-analysis (ISOA): Extensions of an approach to studying short-term developmental processes by analyzing typical patterns. International Journal of Behavioral Development, 36(3), 237-246. http://dx.doi.org/10.1177/0165025412440947

Bergman, L. R., \& Trost, K. (2006). The person-oriented versus the variable-oriented approach: Are they complementary, opposites, or exploring different worlds? Merrill-Palmer Quarterly. Special Issue: Person-Centered and Variable-Centered Approaches to Longitudinal Data, 52(3), 601-632.

Bergman, L. R., \& Vargha, A. (2013). Matching method to problem: A developmental science perspective. European Journal of Developmental Psychology, 10(1), 9-28. http://dx.doi.org/10.1080/17405629.2012.732920

Bergman, L. R., von Eye, A., \& Magnusson, D. (2006). Person-oriented research strategies in developmental psychopathology. In D. Cicchetti \& D. J. Cohen (Eds.), Developmental psychopathology: Theory and method (2nd ed., Vol. 1, pp. 850-888). New York: John Wiley \& Sons.

Block, J. (1971). Lives through time. Berkeley, CA: Bancroft Books.

Bogat, A., von Eye, A., \& Bergman, L. R. (in press). Person-oriented approaches. In D. Cicchetti (Ed.), Developmental Psychopathology (3rd ed.). New York: John Wiley \& Sons.

Boker, S. M. (2002). Consequences of continuity: The hunt for intrinsic properties within parameters of dynamics in psychological processes. Multivariate Behavioral Research, 37(3), 405-422. http://dx.doi.org/10.1207/S15327906MBR3703_5

Boring, E. G. (1950). A history of experimental psychology. New York: AppletonCentury-Crofts.

Bronfenbrenner, U., \& Evans, G. W. (2000). Developmental science in the 21st century: Emerging questions, theoretical models, research designs and empirical findings. Social Development, 9(1), 115-125. http://dx.doi.org/10.1111/1467-9507.00114

Cairns, R. B., Elder, G. H., Jr., \& Costello, E. J. (1996). Developmental Science. Cambridge: Cambridge University Press. http://dx.doi.org/10.1017/CBO9780511571114

Carlson, R. (1971). Where is the person in personality research? Psychological Bulletin, 75(3), 203-219. http://dx.doi.org/10.1037/h0030469

Cicchetti, D. (2006). Development and psychopathology. In D. Cicchetti (Ed.), Developmental psychopathology: Theory and method (2nd ed., Vol. 1, pp. 1-23). New York: John Wiley \& Sons.

Clustan (2005). A comprehensive statistical package for cluster analysis developed by David Wishart. Retrieved from www.clustan.com.

Collins, L. M., \& Wugalter, S. E. (1992). Latent class models for stage-sequential dynamic latent variables. Multivariate Behavioral Research, 27(1), 131-157. http://dx.doi.org/10.1207/s15327906mbr2701_8

Devaney, R. L. (1989). An introduction to chaotic dynamical systems. Redwood City, CA: Addison-Wesley. 
Gibson, W. A. (1959). Three multivariate models: Factor analysis, latent structure analysis, and latent profile analysis. Psychometrika, 24(3), 229-248.

http://dx.doi.org/10.1007/BF02289845

Gottlieb, G. (1996). A systems view of psychobiological development. In D. Magnusson (Ed.), The life-span development of individuals: Behavioral, neurobiological and psychosocial perspectives. A synthesis (pp. 76-103). Cambridge: Cambridge University Press.

Granic, I., \& Hollenstein, T. (2006). A survey of dynamic systems methods for developmental psychopathology. In D. Cicchetti \& D. J. Cohen (Eds.), Developmental psychopathology: Theory and method (2nd ed., Vol. 1, pp. 889-930). New York: John Wiley \& Sons.

Kagan, J. (1998). Galen's prophecy: Temperament in human nature. New York: Basic Books.

Kelso, J. A. (2000). Principles of dynamic pattern formation and change for a science of human behaviour. In L. R. Bergman, R. B. Cairns, L.-G. Nilsson, \& L. Nystedt (Eds.), Developmental science and the holistic approach (pp. 63-84). Mahwah, NJ: Erlbaum.

Lazarsfeld, P. F., \& Henry, N. W. (1968). Latent structure analysis. Boston: Houghton Miffin.

Laursen, B., \& Hoff, E. (2006). Person-centered and variable-centered approaches to longitudinal data. Merrill Palmer Quarterly, 52(3), 377-389. http://dx.doi.org/10.1353/mpq.2006.0029

Lienert, G. A., \& Krauth, J. (1975). Configural frequency analysis as a statistical tool for defining types. Educational and Psychological Measurement, 35(2), 231-238. http://dx.doi.org/10.1177/001316447503500201

Linné, v. C. (1978). Om Jämvikt i naturen. Carmina: Stockholm.

LTA (2013). PROC LCA \& PROC LTA (Version 1.3.0) [Software]. University Park: The Methodology Center, Penn State. Retrieved from http://methodology.psu.edu.

Lundh, L. G., Saboonchi, F., \& Wångby, M. (2008). The role of personal standards in clinically significant perfectionism. A person-oriented approach to the study of patterns of perfectionism. Cognitive Therapy and Research, 32(3), 333-350. http://dx.doi.org/10.1007/s10608-006-9109-7

Magnusson, D. (1988). Individual development from an interactional perspective: A longitudinal study. Hillsdale, NJ, England: Lawrence Erlbaum Associates.

Magnusson, D. (Ed.) (1996). The life-span development of individuals: Behavioral, neurobiological and psychosocial perspectives. Cambridge: Cambridge University Press.

Magnusson, D., \& Törestad, B. (1993). A holistic view of personality: A model revisited. Annual Review of Psychology, 44, 427-452. http://dx.doi.org/10.1146/annurev.ps.44.020193.002235

Meehl, P. E. (1950). Configural scoring. Journal of Consulting Psychology, 14(3), 165171. http://dx.doi.org/10.1037/h0058049

Molenaar, P. C. M. (2004). A manifesto on psychology as idiographic science: Bringing the person back into scientific psychology, this time forever. Measurement: Interdisciplinary Research and Perspectives, 2(4), 201-218.

http://dx.doi.org/10.1207/s15366359mea0204_1 
Mplus (2013). Mplus Version 7.11. Retrieved from www.statmodel.com.

Muthén, B. (2002). Beyond SEM: General latent variable modeling. Behaviormetrika, 29(1), 81-117. http://dx.doi.org/10.2333/bhmk.29.81

Nesselroade, J. R., \& Ford, D. H. (1985). P-technique comes of age: Multivariate, replicated, single-subject designs for research on older adults. Research on Aging, 7(1), 46-80. http://dx.doi.org/10.1177/0164027585007001003

Richters, J. E. (1997). The hubble hypothesis and the developmentalist's dilemma. Development and Psychopathology, 9(2), 193-229. http://dx.doi.org/10.1017/S0954579497002022

ROPstat (2011). A statistical package for nonparametric and person-oriented analyses developed by András Vargha and Peter Bansagi. Retrieved from www.ropstat.com.

SLEIPNER (2002). A statistical package for person-oriented analysis developed by Lars R. Bergman and Bassam El-Khouri. Retrieved from www.psychology.su.se/sleipner/.

Sterba, S. K., \& Bauer, D. J. (2010). Matching method with theory in person-oriented developmental psychopatology research. Development and Psychopathology, 22(2), 239-254. http://dx.doi.org/10.1017/S0954579410000015

Von Eye, A. (1998). Configural frequency analysis program for 32 bit operation systems. Methods of Psychological Research, 3(1), 1-3.

Von Eye, A., \& Bergman, L. R. (2003). Research strategies in developmental psychopathology: Dimensional identity and the person-oriented approach. Development and Psychopathology, 15(3), 553-580.

http://dx.doi.org/10.1017/S0954579403000294

Von Eye, A., \& Pena, G. E. (2004). Configural frequency analysis: The search for extreme cells. Journal of Applied Statistics, 31(8), 981-997.

http://dx.doi.org/10.1080/0266476042000270545 